MATEC Web of Conferences 44, 02075 (2016)

DOI: $10.1051 /$ matecconf $/ 20164402075$

(c) Owned by the authors, published by EDP Sciences, 2016

\title{
Kinematic Analysis of a 3-dof Parallel Machine Tool with Large Workspace
}

\author{
Yan Shi, 2, a , Yan Gao ${ }^{3}$ and Lian $\mathrm{He}^{1} \mathrm{Guo}^{4}$ \\ ${ }^{1}$ Department of Power Engineering, Hebei Institute of Hydraulic and Electric Engineering, Cangzhou, Hebei 061001, China \\ ${ }^{2}$ Water Resources Automation and Informatization Application Technology Research and Development Center of Hebei, Cangzhou, Hebei \\ 061001, China \\ ${ }^{3}$ College of Astronautics, Harbin Institute of Technology, Harbin, Heilongjiang 150000, China \\ ${ }^{4}$ Unit 95927 of the PLA, Cangzhou, Hebei 061736, China
}

\begin{abstract}
Kinematics of a 3-dof (degree of freedom) parallel machine tool with large workspace was analyzed. The workspace volume and surface and boundary posture angles of the 3-dof parallel machine tool are relatively large. Firstly, three dimensional simulation manipulator of the 3-dof parallel machine tool was constructed, and its joint distribution was described. Secondly, kinematic models of the 3-dof parallel machine tool were fixed on, including displacement analysis, velocity analysis, and acceleration analysis. Finally, the kinematic models of the machine tool were verified by a numerical example. The study result has an important significance to the application of the parallel machine tool.
\end{abstract}

\section{Introduction}

Recently, parallel machine tools with limited degrees of freedom (DoF) are widely applied in many situations ${ }^{[1]}$, and lots of scholars carry out research about various parallel machine tools with limited DoF. Among them, parallel machine tools with three degrees of freedom especially the 3-SPR parallel machine tool including spherical joint, prismatic joint, and revolute joint attract more attentions ${ }^{[2-4]}$. For 3-SPR parallel machine tool has less limbs, and its structure is simpler and it can be controlled more easily, it has a great application potential on many occasions no need for six degrees of freedom.

Workspace is an important influencing factor of parallel machine tool performance, it is also one of research focuses of parallel manipulator ${ }^{[5-7]}$. Determination of joint distribution among parallel machine tools with the same structure is specially important, which makes relatively larger workspace. The influence of revolute joint posture to the parallel machine tool performance about 3-SPR parallel machine tool is discussed in reference [8], and two optimum revolute joint postures were derived. On the basis of the above research, a machine tool with optimized joints is chosen as the research object, and its kinematic characteristic is mainly discussed, which lays a foundation for the application of the optimized manipulator.

\section{Introduction of the manipulator}

The 3-SPR manipulator is composed of a moving platform, a fixed platform and three driving limbs. The moving platform and the fixed platform are both equilateral triangle. The three driving limbs have the

a shiyanysu@163.com same structure, and from the bottom to the above is spherical joint, prismatic joint and revolute joint successively. In each limb, the prismatic joint is the driving limb. When the force from the motor is acted on the prismatic joint, the moving platform begins to move. The difference among the three driving limbs is the vector direction of the revolute joint axis. The research result shows that when axes of the three revolute joints intersect on the central point of the moving platform, the volume and the surface and the boundary posture of the manipulator are relatively larger ${ }^{[5]}$. The 3-SPR parallel manipulator studied in this paper is the above mentioned manipulator that the joint distribution is optimized. The three dimensional entity manipulator is shown as Fig. 1, and the revolute joint distribution of the moving platform is shown as Fig. 2. In Fig. 1, $A_{1} A_{2} A_{3}$ is the fixed platform equilateral triangle, and $a_{1} a_{2} a_{3}$ is the moving platform equilateral triangle. In Fig. 2, $\boldsymbol{R}_{1} 、 \boldsymbol{R}_{2} 、 \boldsymbol{R}_{3}$ represent the direction of the revolute vector on points $a_{1}, a_{2}, a_{3}$, the angle between which and $x$ axis of the moving platform is $150^{\circ} 、 90^{\circ} 、 30^{\circ}$, respectively.

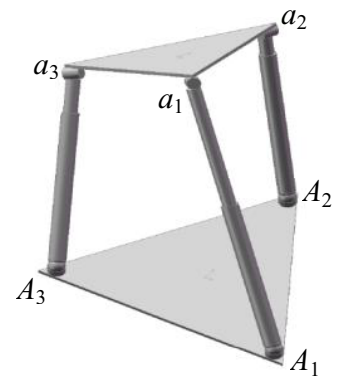

Figure 1. Three dimensional entity manipulator of 3-SPR parallel machine tool 


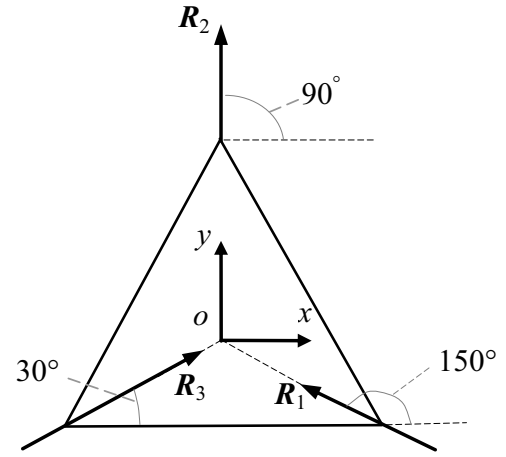

Figure 2. Revolute joint distribution of the moving platform

\section{Kinematic analysis of the manipulator}

\subsection{Displacement analysis}

Let $O-X Y Z$ represent the coordinate of the fixed platform, $o-x y z$ represent the coordinate of the moving platform, and $X_{\mathrm{o}}, Y_{\mathrm{o}}, Z_{\mathrm{o}}$ represent the projection of the central point of the moving platform $o$ on the fixed platform, respectively. Use $X Z X$ Eular angle to express the rotational matrix $\boldsymbol{R}_{u}^{L}$, that is, the angle that the moving platform rotates around the $X$ axis, the $Z_{1}$ axis and the $X_{2}$ axis is $\alpha, \beta, \gamma$, respectively. Among them, $Z_{1}$ axis and $X_{2}$ axis represent the coordinate axis of the middle coordinate when the moving platform rotates for the first time and the second time, respectively. Then, $\boldsymbol{R}_{u}^{L}$ can be expressed as

$$
\begin{aligned}
\mathbf{R}_{u}^{L} & =\left[\begin{array}{ccc}
c \beta & -s \beta \cdot c \gamma & s \beta \cdot s \gamma \\
c \alpha \cdot s \beta & -s \alpha \cdot s \gamma+c \alpha \cdot c \beta \cdot c \gamma & -c \alpha \cdot c \beta \cdot s \gamma-s \alpha \cdot c \gamma \\
s \alpha \cdot s \beta & s \alpha \cdot c \beta \cdot c \gamma+c \alpha \cdot s \gamma & c \alpha \cdot c \gamma-s \alpha \cdot c \beta \cdot s \gamma
\end{array}\right] \\
& =\left[\begin{array}{lll}
x_{l} & y_{l} & z_{l} \\
x_{m} & y_{m} & z_{m} \\
x_{n} & y_{n} & z_{n}
\end{array}\right]
\end{aligned}
$$

Where, $s$ represents sin, $c$ represents cos. It is same in the following sections.

To the studied parallel manipulator, there are the following constraint relations among variables ${ }^{[5]}$

$$
\begin{aligned}
& \sqrt{3} P-Q+2 e+E\left(\sqrt{3} x_{m}-3 x_{l}+\sqrt{3} y_{l}-y_{m}\right) / 2=0 \\
& Q+e-E y_{m}=0 \\
& \sqrt{3} P+Q-2 e+E\left(\sqrt{3} x_{m}+3 x_{l}+\sqrt{3} y_{l}+y_{m}\right) / 2=0
\end{aligned}
$$

Where, $P=X_{o} x_{l}+Y_{o} x_{m}+Z_{o} x_{n}, Q=X_{o} y_{l}+Y_{o} y_{m}+Z_{o} y_{n} . E$ and $e$ represent the circumradius of the fixed platform and the moving platform equilateral triangle, respectively. From Eq. (2) and Eq. (4), it can be derived

$$
\begin{aligned}
& 2 P+E\left(x_{m}+y_{l}\right)=0 \\
& 2 Q-4 e+E\left(3 x_{l}+y_{m}\right)=0
\end{aligned}
$$

From Eq. (3) and Eq. (6), it can be derived

$$
2 e-E\left(x_{l}+y_{m}\right)=0
$$

Substitute Eq. (1) to Eq. (3), Eq. (5) and Eq. (7), respectively, the relations between the three translational variables of the central point $X_{0}, Y_{\mathrm{o}}, Z_{\mathrm{o}}$ and the three rotational variables of the moving platform $\alpha, \beta, \gamma$ can be derived

$$
\begin{aligned}
& -X_{o} s \beta \cdot c \gamma+\left(Y_{o}-E\right)(-s \alpha \cdot s \gamma+c \alpha \cdot c \beta \cdot c \gamma)+ \\
& Z_{o}(s \alpha \cdot c \beta \cdot c \gamma+c \alpha \cdot s \gamma)+e=0 \\
& 2 X_{o} c \beta+\left(2 Y_{o}+E\right) c \alpha \cdot s \beta+2 Z_{o} s \alpha \cdot s \beta-E s \beta \cdot c \gamma=0 \\
& 2 e-E(c \beta-s \alpha \cdot s \gamma+c \alpha \cdot c \beta \cdot c \gamma)=0
\end{aligned}
$$

For the manipulator has three degrees of freedom, there are three independent output variables for the manipulator. Choose one translational variable $X_{\mathrm{o}}$ and two rotational variables $\alpha, \gamma$ as independent output variables, then the relation between the other two translational variables $Y_{\mathrm{o}}, Z_{\mathrm{o}}$ and one rotational variable $\beta$ and the independent output variables can be expressed as $\beta=\arccos K$;

$Y_{o}=\left[\left(3 K c \alpha \cdot c \gamma-2 s \alpha \cdot s \gamma-K^{2} c^{2} \gamma\right) E+2 X_{o} K(K c \gamma+\cot \alpha \cdot s \gamma) /\right.$

$\left.\sqrt{1-K^{2}}+2 X_{o} \sqrt{1-K^{2}} c \gamma-2 e\right] /[s \gamma(c \alpha \cdot \cot \alpha-2 s \alpha)]$;

$Z_{o}=\left[E c \gamma-\left(2 Y_{o}+E\right) c \alpha\right] /(2 s \alpha)-X_{o} K /\left(\sqrt{1-K^{2}} s \alpha\right)$

Where, $K=[(2 e / E+s \alpha \cdot s \gamma) /(1+c \alpha \cdot c \gamma)]$.

The driving limb length of the manipulator can be expressed as

$r_{1}^{2}=X_{o}^{2}+Y_{o}^{2}+Z_{o}^{2}+E^{2}+e^{2}-q E X_{o}+E Y_{o}+q e P-e Q+$

$\mathrm{Ee}\left(q y_{l}-3 x_{l}+q x_{m}-y_{m}\right) / 2$;

$r_{2}^{2}=X_{o}^{2}+\left(Y_{o}-E\right)^{2}+Z_{o}^{2}+e^{2}-2 E e y_{m}+2 e Q ;$

$r_{3}^{2}=X_{o}^{2}+Y_{o}^{2}+Z_{o}^{2}+E^{2}+e^{2}+q E X_{o}+E Y_{o}-q e P-e Q-$

$\mathrm{Ee}\left(q y_{l}+3 x_{l}+q x_{m}+y_{m}\right) / 2$

When $\left(X_{\mathrm{o}}, \alpha, \gamma\right)$ is known, the driving limb length can be derived from Eq. (1), Eq. (9) and Eq. (10).

\subsection{Velocity analysis}

The linear velocity of the central point of the moving platform $o$ can be expressed as

$$
\boldsymbol{v}=\left[\begin{array}{c}
\dot{X}_{o} \\
\dot{Y}_{o} \\
\dot{Z}_{o}
\end{array}\right]=\left[\begin{array}{ccc}
\partial X_{o} / \partial X_{o} & \partial X_{o} / \partial \alpha & \partial X_{o} / \partial \gamma \\
\partial Y_{o} / \partial X_{o} & \partial Y_{o} / \partial \alpha & \partial Y_{o} / \partial \gamma \\
\partial Z_{o} / \partial X_{o} & \partial Z_{o} / \partial \alpha & \partial Z_{o} / \partial \gamma
\end{array}\right]\left[\begin{array}{c}
\dot{X}_{o} \\
\dot{\alpha} \\
\dot{\gamma}
\end{array}\right]=\mathbf{J}_{v} v_{e}
$$

Where, $\mathbf{J}_{v}$ represents the traslational Jacobian matrix of the manipulator ${ }^{[10]}$.

When $X Z X$ Eular angle is adopted, the angular velocity of the moving platform can be expressed as

$$
\boldsymbol{\omega}=\boldsymbol{X} \dot{\alpha}+\boldsymbol{Z}_{1} \dot{\beta}+\boldsymbol{x} \dot{\gamma}
$$

Where,

$$
\begin{aligned}
& \boldsymbol{Z}_{1}=R(X, \alpha)\left[\begin{array}{lll}
0 & 0 & 1
\end{array}\right]^{T}=\left[\begin{array}{lll}
0 & -s \alpha & c \alpha
\end{array}\right]^{T}, \\
& \boldsymbol{x}=\left[\begin{array}{lll}
x_{l} & x_{m} & x_{n}
\end{array}\right]^{T}=\left[\begin{array}{lll}
c \beta & c \alpha \cdot s \beta & s \alpha \cdot s \beta
\end{array}\right]^{T} \\
& \boldsymbol{\omega}=\left[\begin{array}{ccc}
1 & 0 & c \beta \\
0 & -s \alpha & c \alpha \cdot s \beta \\
0 & c \alpha & s \alpha \cdot s \beta
\end{array}\right]\left[\begin{array}{c}
\dot{\alpha} \\
\dot{\beta} \\
\dot{\gamma}
\end{array}\right]=
\end{aligned}
$$$$
\left[\begin{array}{ccc}
1 & 0 & c \beta \\
0 & -s \alpha & c \alpha \cdot s \beta \\
0 & c \alpha & s \alpha \cdot s \beta
\end{array}\right]\left[\begin{array}{ccc}
\partial \alpha / \partial X_{o} & \partial \alpha / \partial \alpha & \partial \alpha / \partial \gamma \\
\partial \beta / \partial X_{o} & \partial \beta / \partial \alpha & \partial \beta / \partial \gamma \\
\partial \gamma / \partial X_{o} & \partial \gamma / \partial \alpha & \partial \gamma / \partial \gamma
\end{array}\right]\left[\begin{array}{c}
\dot{X}_{o} \\
\dot{\alpha} \\
\dot{\gamma}
\end{array}\right]
$$

$$
\begin{aligned}
& =\left[\begin{array}{ccc}
1 & 0 & c \beta \\
0 & -s \alpha & c \alpha \cdot s \beta \\
0 & c \alpha & s \alpha \cdot s \beta
\end{array}\right]\left[\begin{array}{ccc}
0 & 1 & 0 \\
\partial \beta / \partial X_{o} & \partial \beta / \partial \alpha & \partial \beta / \partial \gamma \\
0 & 0 & 1
\end{array}\right]\left[\begin{array}{c}
\dot{X}_{o} \\
\dot{\alpha} \\
\dot{\gamma}
\end{array}\right] \\
& =\mathbf{J}_{\omega} \boldsymbol{v}_{e}
\end{aligned}
$$


Where, $\mathbf{J}_{\omega}$ represents the rotational Jacobian matrix of the manipulator ${ }^{[10]}$.

The velocity of the driving limb can be expressed as

$$
\boldsymbol{v}_{\boldsymbol{r}}=\left[\begin{array}{lll}
\dot{r}_{1} & \dot{r}_{2} & \dot{r}_{3}
\end{array}\right]^{T}=\left[\begin{array}{ll}
\boldsymbol{\delta}_{1}^{T} & \left(\boldsymbol{e}_{1} \times \boldsymbol{\delta}_{1}\right)^{T} \\
\boldsymbol{\delta}_{2}^{T} & \left(\boldsymbol{e}_{2} \times \boldsymbol{\delta}_{2}\right)^{T} \\
\boldsymbol{\delta}_{3}^{T} & \left(\boldsymbol{e}_{3} \times \boldsymbol{\delta}_{3}\right)^{T}
\end{array}\right]_{3 \times 6}\left[\begin{array}{c}
\boldsymbol{v} \\
\boldsymbol{\omega}
\end{array}\right]
$$

Where, $\boldsymbol{e}_{i}$ represents the vector from the central point to the vertex of the moving platform, $\boldsymbol{\delta}_{i}$ represents the unit vector of the moving platform $(i=1,2,3)$. When $\left(X_{\mathrm{o}}, \alpha, \gamma\right)$ and $\left(\dot{X}_{o}, \dot{\alpha}, \dot{\gamma}\right)$ are known, the velocity of the driving limbs can be derived from Eq. (11) to Eq. (13).

\subsection{Acceleration analysis}

The acceleration of the driving limbs can be expressed as

$$
\boldsymbol{a}_{\boldsymbol{r}}=\left[\begin{array}{c}
\ddot{r}_{1} \\
\ddot{r}_{2} \\
\ddot{r}_{3}
\end{array}\right]=\left[\begin{array}{cc}
\boldsymbol{\delta}_{1}{ }^{T} & \left(\boldsymbol{e}_{1} \times \boldsymbol{\delta}_{1}\right)^{T} \\
\boldsymbol{\delta}_{2}{ }^{T} & \left(\boldsymbol{e}_{2} \times \boldsymbol{\delta}_{2}\right)^{T} \\
\boldsymbol{\delta}_{3}{ }^{T} & \left(\boldsymbol{e}_{3} \times \boldsymbol{\delta}_{3}\right)^{T}
\end{array}\right]_{3 \times 6} \boldsymbol{A}+\boldsymbol{V}^{\boldsymbol{T}}\left[\begin{array}{c}
\boldsymbol{H}_{1} \\
\boldsymbol{H}_{2} \\
\boldsymbol{H}_{3}
\end{array}\right] \boldsymbol{V}
$$

Where, $\boldsymbol{A}=\left[\begin{array}{ll}\boldsymbol{a} & \boldsymbol{\varepsilon}\end{array}\right]^{T}, \boldsymbol{a}=\left[\begin{array}{lll}a_{x} & a_{y} & a_{z}\end{array}\right]^{T}$ represents the linear acceleration of the central point of the moving platform, $\boldsymbol{\varepsilon}=\left[\begin{array}{lll}\varepsilon_{x} & \varepsilon_{y} & \varepsilon_{z}\end{array}\right]^{T}$ represents the angular acceleration of the moving platform, and $\boldsymbol{V}=\left[\begin{array}{ll}\boldsymbol{v} & \boldsymbol{\omega}\end{array}\right]^{T}$, $\boldsymbol{v}=\left[\begin{array}{lll}v_{x} & v_{y} & v_{z}\end{array}\right]^{T}$ represents the linear velocity of the central point of the moving platform, $\boldsymbol{\omega}=\left[\begin{array}{lll}\omega_{x} & \omega_{y} & \omega_{z}\end{array}\right]^{T}$ represents the angular velocity of the moving platform.

$$
\begin{aligned}
& \boldsymbol{H}_{i}=\frac{1}{r_{i}}\left[\begin{array}{cc}
\boldsymbol{E}_{3 \times 3} & -\hat{\boldsymbol{e}}_{i} \\
\hat{\boldsymbol{e}}_{i} & -\hat{\boldsymbol{e}}_{i}{ }^{2}
\end{array}\right]_{6 \times 6}-\frac{1}{r_{i}}\left[\begin{array}{c}
\boldsymbol{\delta}_{i} \\
\boldsymbol{e}_{i} \times \boldsymbol{\delta}_{i}
\end{array}\right]\left[\begin{array}{ll}
\boldsymbol{\delta}_{i}^{T} & \left(\boldsymbol{e}_{i} \times \boldsymbol{\delta}_{i}\right)^{T}
\end{array}\right] \\
& +\left[\begin{array}{ll}
\mathbf{0}_{3 \times 3} & \mathbf{0}_{3 \times 3} \\
\mathbf{0}_{3 \times 3} & \hat{\boldsymbol{e}}_{i} \hat{\boldsymbol{\delta}}_{i}
\end{array}\right]_{6 \times 6}, \quad(i=1,2,3) \\
& \hat{\boldsymbol{e}}_{i}=\left[\begin{array}{ccc}
0 & -e_{i z} & e_{i y} \\
e_{i z} & 0 & -e_{i x} \\
-e_{i y} & e_{i x} & 0
\end{array}\right], \hat{\boldsymbol{\delta}}_{i}=\left[\begin{array}{ccc}
0 & -\delta_{i z} & \delta_{i y} \\
\delta_{i z} & 0 & -\delta_{i x} \\
-\delta_{i y} & \delta_{i x} & 0
\end{array}\right]
\end{aligned}
$$

The relation of the acceleration of the moving platform $\boldsymbol{A}$ and the acceleration of the independent variables $\left(\ddot{X}_{o} 、 \ddot{\alpha} 、 \ddot{\gamma}\right)$ can be expressed as

$$
\begin{gathered}
\boldsymbol{A}=\left[\begin{array}{l}
\boldsymbol{J}_{\boldsymbol{v}} \\
\boldsymbol{J}_{\boldsymbol{\omega}}
\end{array}\right]\left[\begin{array}{c}
\ddot{X}_{o} \\
\ddot{\alpha} \\
\ddot{\gamma}
\end{array}\right]+\left[\begin{array}{lll}
\dot{X}_{o} & \dot{\alpha} & \dot{\gamma}
\end{array}\right]^{T}\left[\begin{array}{c}
\boldsymbol{H}_{\boldsymbol{v}} \\
\boldsymbol{H}_{\boldsymbol{\omega}}
\end{array}\right]\left[\begin{array}{c}
\dot{X}_{o} \\
\dot{\alpha} \\
\dot{\gamma}
\end{array}\right] \\
\boldsymbol{H}_{\boldsymbol{v} i}=\left[\begin{array}{lll}
\partial \boldsymbol{J}_{v i 1} / \partial X_{o} & \partial \boldsymbol{J}_{v i 2} / \partial X_{o} & \partial \boldsymbol{J}_{v i 3} / \partial X_{o} \\
\partial \boldsymbol{J}_{v i 1} / \partial \alpha & \partial \boldsymbol{J}_{v i 2} / \partial \alpha & \partial \boldsymbol{J}_{v i 3} / \partial \alpha \\
\partial \boldsymbol{J}_{v i 1} / \partial \gamma & \partial \boldsymbol{J}_{v i 2} / \partial \gamma & \partial \boldsymbol{J}_{v i 3} / \partial \gamma
\end{array}\right], \\
\text { Where, } \boldsymbol{H}_{\omega i}=\left[\begin{array}{lll}
\partial \boldsymbol{J}_{\omega i 1} / \partial X_{o} & \partial \boldsymbol{J}_{\omega i 2} / \partial X_{o} & \partial \boldsymbol{J}_{\omega i 3} / \partial X_{o} \\
\partial \boldsymbol{J}_{\omega i 1} / \partial \alpha & \partial \boldsymbol{J}_{\omega i 2} / \partial \alpha & \partial \boldsymbol{J}_{\omega i 3} / \partial \alpha \\
\partial \boldsymbol{J}_{\omega i 1} / \partial \gamma & \partial \boldsymbol{J}_{\omega i 2} / \partial \gamma & \partial \boldsymbol{J}_{\omega i 3} / \partial \gamma
\end{array}\right]
\end{gathered}
$$

$$
(i=1,2,3)
$$

When $\left(X_{0}, \alpha, \gamma\right),\left(\dot{X}_{o} 、 \dot{\alpha} 、 \dot{\gamma}\right)$ and $\left(\ddot{X}_{o} 、 \ddot{\alpha} 、 \ddot{\gamma}\right)$ are known, the acceleration of the driving limbs can be derived from Eq. (16) to Eq. (18).

\section{Numerical experiment}

Suppose the side length of the moving platform equilateral triangle is $50 \mathrm{~cm}$, the side length of the fixed platform equilateral triangle is $100 \mathrm{~cm}$, and the initial displacement of the independent output variables $X_{o s}=10 \mathrm{~cm}, \alpha_{s}=-5^{\circ}, \gamma_{s}=15^{\circ}$, the initial velocity of the independent output variables $v_{X o s}=1 \mathrm{~cm} / \mathrm{s}, v_{\alpha s}=-0.5^{\circ} / \mathrm{s}, v_{\gamma s}=$ $0.2 \%$, the initial acceleration of the independent output variables $a_{X o s}=0.1 \mathrm{~cm} / \mathrm{s}^{2}, a_{\alpha s}=-0.01^{\circ} / \mathrm{s}^{2}, a_{\gamma s}=0.01^{\circ} / \mathrm{s}^{2}$.

Under the above conditions, the velocity of the moving platform is shown as Fig. 3, and the linear velocity of the central point of the moving platform is shown as Fig. 3a, the angular velocity of the moving platform is shown as Fig. $3 \mathrm{~b}$. The acceleration of the moving platform is shown as Fig. 4, and the linear acceleration of the central point of the moving platform is shown as Fig. 4a, the angular acceleration of the moving platform is shown as Fig. 4b. The kinematic solving results are shown as Fig. 5, and the displacement curve of the driving limbs is shown as Fig. 5a, the velocity curve of the driving limbs is shown as Fig. 5b, the acceleration curve of the driving limbs is shown as Fig. 5c.

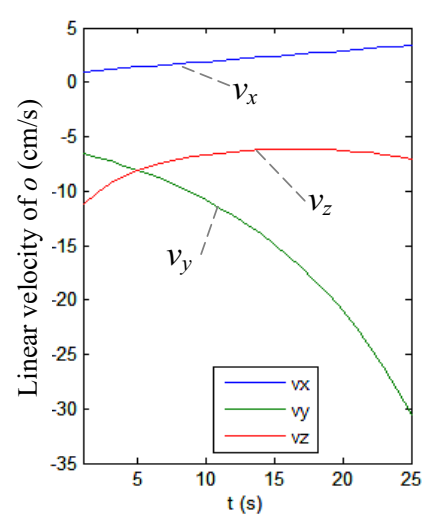

Figure 3a. Linear velocity of the central point of the moving platform

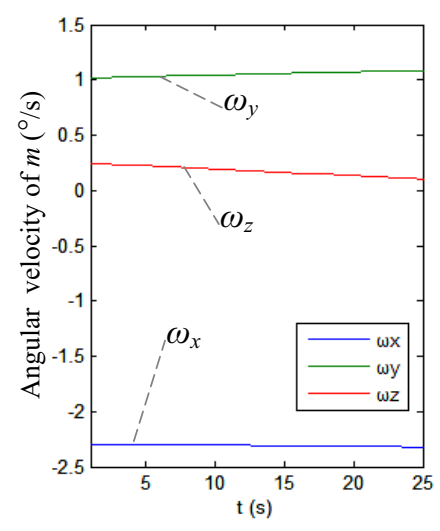

Figure $3 \mathrm{~b}$. Angular velocity of the moving platform 


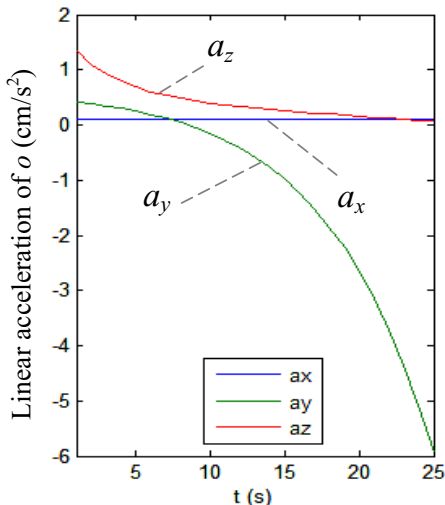

Figure 4a. Linear acceleration of the central point of the moving platform

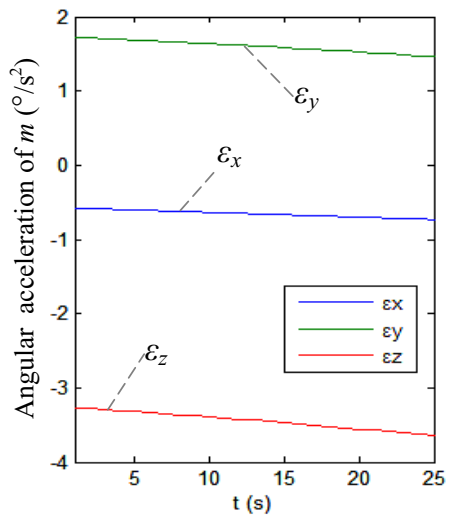

Figure 4b. Angular acceleration of the moving platform

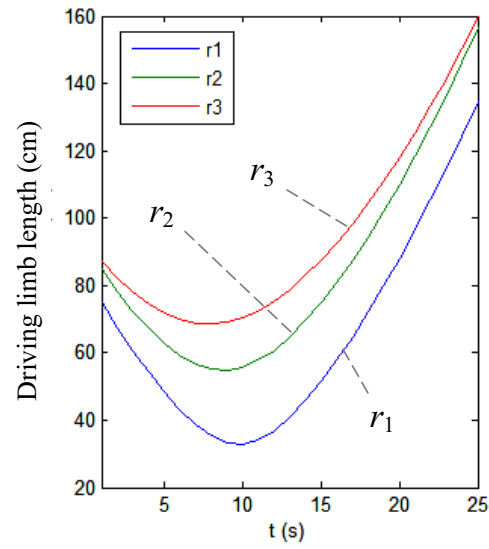

Figure 5a. Length curve of the driving limbs

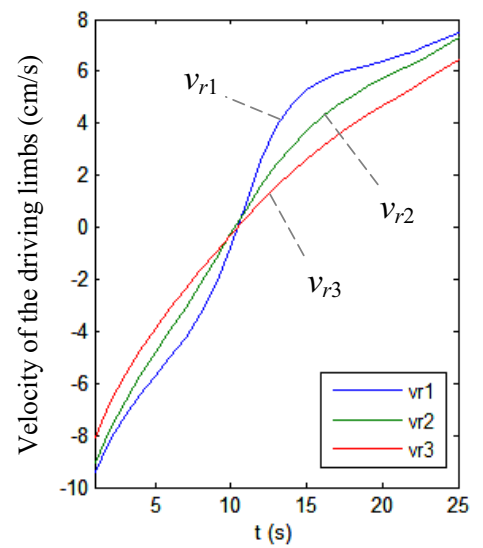

Figure $5 b$. Velocity curve of the driving limbs

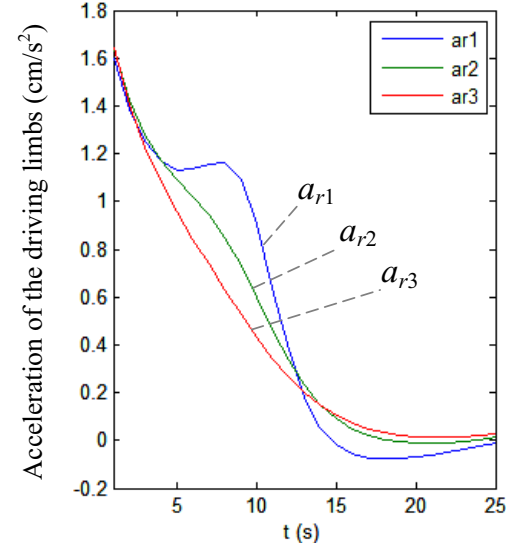

Figure 5c. Acceleration curve of the driving limbs

\section{Conclusion}

For the 3-SPR parallel manipulator, when axes of the three revolute joints intersect on the central point of the moving platform, the volume and the surface and the boundary posture of the manipulator are not only relatively larger, the kinematic model is also not complex. Therefore, the 3-SPR parallel manipulator with this joint distribution has more practical value. The constructed kinematic model lays the foundation for the statics, dynamics and stiffness analysis of this parallel machine tool, it also provides the instruction for the displacement control, velocity control and acceleration control during the application of the parallel machine tool.

\section{Acknowledgement}

This research was financially supported by Higher Education Institution Science and Technology Research Project of Hebei Province (QN2015158).

\section{References}

1. Y. Lu, B. Hu. Development evaluation of limitedDOF parallel manipulators [J]. Journal of Yanshan University, 2011, 35(5): 377-384.

2. N. M. Rao, K. M. Rao. Multi-position dimensional synthesis of a spatial 3-RPS parallel manipulator [J]. ASME Journal of Mechanical Design, 2006, 128(4): 815-819.

3. X. J. Liu, J. S. Wang, G. Pritschow. A new family of spatial 3-DoF fully-parallel manipulators with high rotational capability [J]. Mechanism and Machine Theory, 2005, 40(4): 475-494.

4. R. Sellaouti, F. B. Ouezdou. Design and control of a 3DOFs parallel actuated mechanism for biped application [J]. Mechanism and Machine Theory, 2005, 40(12): 1367-1393.

5. X. P. Hu, B. Li, W. S. Zhao. Research on workspace analysis of a 3-PRS-PP based parallel kinematic machine [J]. Combined Machine Tool and Automatic Machining Technology, 2005, (5):12-13.

6. Y. Lu, Y. Shi, B. Hu. Solving Reachable Workspace of Some Parallel Manipulators by CAD Variation Geometry [J]. Proceedings of the Institution of 
Mechanical Engineers, Part C, Journal of Mechanical Engineering Science, 2008, 222(9):1773-1781

7. H. Li, Y. R. Zhang, D. X. Wang. Comparative analysis of optimization algorithms in workspace optimization of parallel mechanisms [J]. Journal of Mechanical Engineering, 2010, 46(13): 61-67.

8. Y. Shi, Y. Lu. Analysis about workspace of 3-SPR parallel manipulator influenced by the joints' distribution [J]. Journal of Yanshan University, 2008, 32(4): 304-310.

9. Z. Huang, Y. S. Zhao, T. S. Zhao. Advanced Spatial Mechanism [M]. Beijing: Higher Education Press, 2006: 119.

10. Y. Lu, Y. Shi, J. P. Yu. Kinematic analysis of limited-DOF parallel manipulators based on translational/rotational Jacobian and Hessian Matrices [J]. Robotica, 2009, 27(7): 971-980. 